19 Revue d'histoire du XIXe siècle

Société d'histoire de la révolution de 1848 et des

révolutions du XIXe siècle

42 | 2011

La souveraineté populaire

\title{
Introduction : De la souveraineté
}

\section{Michèle Riot-Sarcey}

URL : http://journals.openedition.org/rh19/4100

DOI : $10.4000 /$ rh 19.4100

ISSN : $1777-5329$

Éditeur

La Société de 1848

Édition imprimée

Date de publication : 15 juillet 2011

Pagination : 7-17

ISSN : 1265-1354

Référence électronique

Michèle Riot-Sarcey, «Introduction : De la souveraineté », Revue d'histoire du XIXe siècle [En ligne], 42 |

2011, mis en ligne le 01 août 2011, consulté le 10 décembre 2020. URL : http://

journals.openedition.org/rh19/4100; DOI : https://doi.org/10.4000/rh19.4100 


\section{MICHÈLE RIOT-SARCEY}

\section{De la souveraineté}

Désormais, l'idée de souveraineté est si étroitement associée au pouvoir politique que la recherche historique qui s'y rapporte tend à limiter sa quête aux moments insurrectionnels et aux phases révolutionnaires. Dans ce numéro, La souveraineté populaire en Europe. Expériences et normalisations (1800-1848), afin d'atteindre la connaissance des manifestations «souveraines» du peuple qui se présentent sous les aspects les plus informels, nous avons délibérément écarté l'examen des périodes révolutionnaires proprement dites, pour nous concentrer sur quelques fragments, ou incises, d'expressions souveraines. Dans la première moitié du XIX ${ }^{e}$ siècle, en effet, en l'absence de droits politiques, dans plusieurs pays européens, le peuple recourt à des formes de contestation et de prises de pouvoir très singulières : il y exprime parfois une aspiration à la liberté en tentant d'instaurer sa propre loi, à la manière du royal souverain d'antan.

Le choix de s'attarder sur des pratiques souveraines s'est d'autant plus imposé que le concept à lui seul ne suffit pas à rassembler la diversité des expressions concrètes de l'idée de souveraineté. De Palerme à Dublin, l'aspiration à la souveraineté populaire se déploie diversement : dans des lieux publics où le peuple rassemblé déstabilise l'ordre existant; dans des endroits improbables où le rituel l'emporte sur le discours politique; plus classiquement, dans l'espace de travail, par l'appropriation de l'outil de production. Le souverain populaire se donne à voir, précisément dans cette période d'après la Révolution française, jusqu'à se confondre, par exemple, en Irlande et en Espagne, avec le patriotisme national, dans un mouvement d'émancipation à l'encontre de la tutelle royale.

Au cours de ces deux derniers siècles, l'idée de souveraineté s'est transformée, sans infléchir le sens courant de l'expression qui la désigne : comme si la linéarité du mot suffisait à faire entendre son contenu. Clairement explicitée à l'aube de la modernité, au cours du temps, la signification du souverain a perdu sa forme populaire en se logeant dans une représentation à distance du réel. La présence active et massive du souverain fut alors vidée de ses références et, peu à peu, dépossédée de son pouvoir, y compris celui de contrôler ses représentants. 
Et pourtant, le concept n'a cessé de ressurgir, comme si l'idée ${ }^{1}$ dans laquelle il s'inscrivait restait intacte. Inachevée, en quelque sorte.

Parce qu'il nous paraissait important de comprendre comment la notion de souveraineté avait pu perdre sa valeur révolutionnaire, il nous a semblé nécessaire de retrouver les traces des multiples tentatives dont elle avait fait l'objet. Expériences éphémères, fragmentaires d'une souveraineté politique décalée de l'exercice des pouvoirs d'État, réduites à de petits arrangements avec les interdits et les impossibilités du temps, ces expériences n'en restent pas moins l'expression d'un besoin collectif de souveraineté qui, en «affirmant ses droits» dit avoir «la loi de son côté »². Un moment entrevue, l'idée de liberté accompagne ces manifestations qui marquent la volonté populaire de rester maîtresse de l'espace social qui est le sien.

Délestée des exigences populaires, la souveraineté contemporaine a perdu néanmoins jusqu'au souvenir de ces formes passées, lesquelles, commodément, ont été classées dans la catégorie des illusions ou des utopies. Et pourtant ces fulgurances, même normalisées, éclairent l'attachement des populations à leur pratique concrète. Or, la présence active des catégories populaires est aujourd'hui subsumée par une représentation qui, très tôt, fut déliée de l'action du peuple ${ }^{3}$ dont on sollicite, ponctuellement, à partir de 1848, la délégation de pouvoir, jusqu'à lui faire oublier l'exercice de son usage.

Dès le XVIII e siècle, Rousseau, on le sait, élaborait le sens du souverain moderne. «À l'instant que le peuple est légitimement assemblé en corps souverain, toute juridiction du gouvernement cesse, la puissance exécutive est suspendue, et la personne du dernier citoyen est aussi sacrée et inviolable que celle du premier magistrat, parce qu'où se retrouve le représenté, il n'y a plus de représentant ${ }^{4}$. La question des limites de la représentation n'a pas seulement été imaginée par Rousseau, elle s'est concrètement posée, à chaque instant, dans des lieux les plus divers, tout au long de la Révolution française : des sections aux districts, de la Commune de Paris aux assemblées, jusqu'aux pétitions, qui sont alors présentées physiquement par les requérants. Pendant cette période agitée, la tension entre la représentation et l'exercice du pouvoir s'est manifestée de manière permanente. La présence souveraine est au cœur des enjeux révolutionnaires. C'est pourquoi les autorités du XIX siècle, partisanes ou non de la souveraineté populaire, continuent de dialoguer avec Rousseau, institué penseur accompli de l'idée de souveraineté. "La souveraineté ne peut être représentée, par la même raison qu'elle ne peut être aliénée; elle consiste essentiellement dans la volonté générale, et la volonté ne se

1. La diversité des formes de souveraineté nous amène logiquement à distinguer l'idée du souverain, dont les sens et les applications sont multiples, du concept de souverain, lequel à la fois réduit et rassemble les significations dans l'acception unique d'un sens commun à une époque donnée.

2. Claude Lefort, La complication. Retour sur le communisme, Paris, Fayard, 1999, p. 53.

3. Très longtemps pensé au masculin. p. 132 .

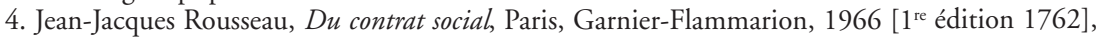


représente point, elle est la même, ou elle est autre. Il n'y a point de milieu "5. Quant à la volonté générale, selon le philosophe des Lumières, elle est la loi fondamentale dans laquelle s'inscrit le pacte social. Expression du souverain, elle n'implique pas l'unanimité; au contraire, son maître mot est pluralité, sans laquelle la liberté n'existe pas.

L'idée de souveraineté, ainsi déclinée, reste abstraite; néanmoins, dans la pratique souveraine, pendant les périodes révolutionnaires, elle prend forme. Dès le Directoire, on le sait, la représentation du souverain, aussi populaire soit-il, s'impose. Néanmoins, si l'idée des représentants est moderne, selon les théoriciens du politique - de Benjamin Constant à Alexis de Tocqueville -, les contemporains de la première moitié du siècle y consentent sans vouloir écarter les pratiques d'une souveraineté héritée de l'esprit des Lumières. Au cours de son enquête auprès des canuts lyonnais, Villermé a d'ailleurs salué une population "intelligente», capable d'échanger des idées modernes. Il la juge plus "avancée dans la civilisation", qu'on ne le croyait à l'époque : "Ils soutenaient d'ailleurs, écrit-il, que l'homme, qui a su se créer des richesses par son industrie, doit en avoir la jouissance entière pendant toute sa vie $»^{6}$. En effet, la volonté d'exercer sa liberté s'exprime différemment, mais toujours avec insistance, dans la plupart des pays européens. À défaut de détenir le souverain pouvoir dans l'espace public, la maîtrise de l'espace de vie, dans lequel l'intervention libre est encore possible, reste la forme de l'expression souveraine, un temps entrevue. Les manifestations populaires, en Angleterre ou en Irlande, disent le besoin d'exprimer leur propre loi, de manière suffisamment vigoureuse pour faire craindre le pire aux classes dirigeantes. Des manifestations ouvrières, retrouvées par Edward P. Thomson, aux sociétés secrètes comme les Impénétrables qui, en France, de 1815 à 1817, diffusent clandestinement leur manifeste visant à rétablir les droits du peuple souverain en France, en Italie et en Espagne ${ }^{7}$, chacun à sa manière retrouve des accents d'autonomie qui rappellent la volonté d'être libre. Souverainement. Appartenant à des catégories minorées, affectées par un sentiment national, encouragé par des leaders (de O'Connell à Mazzini), dirigé ou conseillé par des élites libérales, le peuple en masse, ou en petits groupes plus ou moins autonomes, est alors largement indéterminé. Héritier de la Révolution tout

5. Idem, p. 134

6. Louis-René Villermé, Tableau de l'état physique et moral des ouvriers employés dans les manufactures de coton, de laine et de soie, Paris, Études et documentation internationales, 1989 [1 $1^{\mathrm{re}}$ édition 1840], p. 316.

7. «Quoi! L'État le plus florissant de l'Europe, le peuple le plus courageux et le plus énergique verrait à chaque instant un abîme se creuser sous ses pas. Son commerce anéanti, son industrie absorbée, ses richesses passées en des mains étrangères, ses braves défenseurs humiliés et réduits à la mendicité, sa liberté détruite, son existence compromise, en un mot tout espoir de bonheur enlevé sans retour. Ah! Nous perdrions à jamais le droit de nous plaindre de nos oppresseurs, si nous étions assez lâches pour ne pas leur infliger le châtiment qu’ont mérité leurs forfaits, n’est-ce pas autoriser le crime que de le laisser impuni? ", extrait du Manifeste des Impénétrables, 1816, cité in Laurent Nagy, Le romantisme en action, thèse de doctorat en histoire sous la direction de Michèle Riot-Sarcey, Université Paris 8 - Saint-Denis, 2006. 
en étant sous influence des pensées libérales ou utopiques, il exprime cependant très nettement son attachement à la souveraineté par la volonté d'imposer sa loi contre l'ordre existant, au nom du droit naturel.

De la souveraineté concrète à la souveraineté rêvée, l'idée vagabonde au cours de la première moitié du XIX ${ }^{\mathrm{e}}$ siècle. Comme si le souverain ne pouvait se défaire de son expérience populaire qui s'exprime, ici et là, par des groupes soucieux de répondre aux espérances d'un monde promis mais non encore établi; un monde dont les révolutions de 1848 donnent la configuration la plus aboutie, en s'incarnant, en France particulièrement, dans l'idée de République démocratique et sociale. Son origine immémoriale se décline alors dans tous les temps : de la naissance du christianisme à la chute du souverain royal, de la Grèce antique à la France révolutionnaire. À la manière d'un Constantin Pecqueur qui se réfere aux premiers disciples de Jésus-Christ, auquel il attribue le slogan saint-simonien : "De chacun selon ses forces et ses aptitudes; à chacun selon ses besoins ${ }^{8}$. De son point de vue, république, socialisme, communisme ne font qu'un, au sein d'une souveraineté bien pensée. «L'histoire n'est pas obligée de se répéter servilement [...]. On peut obtenir une nouvelle nuit du 4 août par la voie pacifique et par la seule vertu de la manifestation souveraine du peuple, désormais conscient de son droit et de sa force; on peut obtenir la déchéance des derniers privilèges, comme on désarme quelques hommes d'iniquité, lorsqu'ils se voient circonvenus de tous côtés par une force et une démonstration accablantes " ${ }^{9}$. Avec Pecqueur, nous saisissons l'impact du réveil récurrent de l'idée de souveraineté, reformulée dans une perspective nouvelle. Mais pour en comprendre la portée, l'historien se doit d'être attentif au sens, c'est-à-dire à l'historicité de son énonciation, afin de se donner les moyens d'appréhender les conditions de réapparition d'un même mot dans une situation temporelle totalement éloignée de l'origine de son émergence. «Chaque fois que l'origine se manifeste, écrit justement Walter Benjamin dans son étude sur la période baroque, on voit se définir la figure dans laquelle une idée ne cesse de se confronter au monde historique, jusqu'à ce qu'elle se trouve achevée dans la totalité de son histoire ${ }^{10}$. À l'attention fixée sur le sens, il nous faut ajouter la nécessité d'approfondissement de la pratique des contemporains. En effet, la plupart des idées proches de l'idéal s'inscrivent parfois dans le quotidien des luttes à partir d'un point de vue très éloigné du sens commun de souveraineté qui, dans le long XIX ${ }^{e}$ siècle, se limite à la dimension politique du gouvernement des hommes. En ce dernier sens, la souveraineté, au XIX siècle, n'est plus un acte populaire, elle est représentée. Sous cette forme, elle participe de la modernité. Étroitement liée aux forces productives et aux catégories sociales

8. Le Salut du Peuple. Journal de la science sociale, Paris, J. Ballard, 1849-1850, introduction p. 4.

9. Idem, p. 6.

10. Walter Benjamin, Origine du drame baroque allemand, collection Champs, Paris, Flammarion, 1985 (1édition allemande 1928), p. 44. 
qui les engendrent, la modernité, ainsi conçue, est annonciatrice de progrès humain, dans un temps présent vécu comme incarnation du futur, sinon hérö̈que, pour le moins prometteur de bonheur humain. La modernité du $\mathrm{XIX}^{\mathrm{e}}$ siècle porte en elle cette puissance téléologique, aujourd'hui mise en cause.

Or, la première moitié du XIX siècle ne peut se comprendre avec le même concept qui, traditionnellement, caractérise le long XIX ${ }^{e}$ siècle. Si les découvertes techniques - à l'origine de la révolution industrielle - participent de la philosophie du progrès, le progrès social, indissociable de la modernité, est au centre des mobilisations populaires. Et, à l'encontre de cet horizon d'attente, le progrès est repoussé à des lendemains toujours plus éloignés des réalités. En conséquence, deux directions discordantes voient le jour dans l'aperception de la philosophie du progrès par les contemporains. Les uns, hommes libérés des autorités tutélaires, selon la conception d'Emmanuel Kant ${ }^{11}$, pensent le progrès dans un devenir hiérarchique, les autres cherchent à conquérir une liberté qui, jusqu'alors, leur a échappé. Autant cette prospective pouvait rester à l'état de principe, à l'époque des Lumières, autant devait-elle se concrétiser dès les premières décennies du XIX' siècle. Car, pour la première fois, le peuple devient visible massivement et se pose en candidat au mieux-être. Tandis que le peuple cherche à s'inscrire dans l'histoire, en réclamant ses droits à la liberté, les autorités morales et politiques s'évertuent à créer les conditions de son adaptation aux nouvelles formes de l'existence industrielle. Dans cette vision du progrès, l'asservissement de la nature entrâne immanquablement l'exploitation de l'homme par l'homme, processus qui conduit les saint-simoniens - pourtant grands promoteurs de la révolution industrielle -, à croire qu'ils assistent à son extinction ${ }^{12}$.

Notons cependant que les années qui suivent la chute du Premier Empire sont davantage connues pour l'influence des doctrines utopiques - de SaintSimon à Fourier et d'Owen à Cabet -, que par l'esprit de liberté qui se déploie bien au-delà de la jeunesse romantique. Le peuple, érigé en héros dans la littérature, n'en est pas moins candidat à la souveraineté; une souveraineté espérée qui, d'abord se décline en termes de droit et de liberté. À défaut, il s'exprime par l'insurrection et la révolte : de la France à l'Angleterre, de la Sicile à la Grèce. Le rêve d'une autre société travaille particulièrement la période qui précède les révolutions de 1848 . Un rêve tendu vers l'avenir où le progrès humain demeure incompatible avec l'exploitation de l'homme par l'homme. Tandis que la modernité réelle est parvenue à enfermer l'individu dans des catégories inférieures à celle des hommes libres, l'affranchissement

11. Selon Kant, «Les Lumières, c'est la sortie de l'homme hors de l'état de tutelle dont il est lui-même responsable» et, ajoute-t-il, "pour les Lumières, il n'est rien requis d'autre que la Liberté». Emmanuel Kant, Qu'est-ce que les Lumières?, Paris, Garnier-Flammarion, 1991 (1 ${ }^{\text {re }}$ édition 1784), p. 43 et 45.

12. Cf. Exposition de la doctrine saint-simonienne, 1829-1830. 
«de la classe la plus nombreuse et la plus pauvre $»^{13}$ est au cœur des préoccupations du moment. Comme si les promesses annoncées par la Révolution française devaient voir le jour et s'appliquer ici et maintenant, tant l'expansion de la misère, partout en Europe, est déplorée. Elle suscite les craintes les plus alarmistes auprès de ceux qui redoutent la déstabilisation de la société.

Eugène Buret, parmi bien d'autres réformateurs, après avoir fustigé la misère en France, en Angleterre, en Irlande et dans divers pays, salue les initiatives de ceux qu'il refuse de nommer utopistes : «L'école saint-simonienne qui a jeté un si vif éclat lorsqu'elle faisait la critique de notre état social, a provoqué des discussions sérieuses [...]. Une autre école sociale qui procède aussi d'un novateur de génie, celle de Charles Fourier, plus complète et plus étendue que la première, prétend avoir trouvé la solution définitive et entière de tous les problèmes de sociabilité. Elle a prononcé une grande et puissante parole, le mot d'association, et reconnaissant, avec une admirable intelligence, que l'hostilité des intérêts économiques, la séparation du capital et du travail étaient la véritable cause de tous nos maux, elle a conclu que le seul moyen de les faire cesser, était de réunir, dans une intime solidarité, par l'association, les intérêts divergents $»^{14}$. Les projets de réformes émanent de toutes parts pour tenter de répondre à l'explosion du peuple en lutte, en visant à réguler ses manifestations intempestives, tout en moralisant une catégorie sociale dont on fustige les excès. Et si la lutte de classe reste étrangère aux réformateurs du temps, la réalité de l'exploitation au quotidien ravive la mémoire révolutionnaire et engendre une floraison de réappropriations souveraines, des sociétés de secours mutuel aux associations, licites ou illicites.

Au sein de cette modernité spécifique, précisément, quand la "sainte canaille» s'éveille ponctuellement à «l'immortalité», paradoxalement l'idée de souveraineté populaire, à nouveau en mouvement dans les années 1830 , s'efface ou se normalise, au profit d'une représentation d'un peuple que la plupart des vainqueurs des révolutions anglaises et françaises jugent incapable d'exercer directement un pouvoir souverain.

Benjamin Constant, le premier, l'explicite en théorisant la distinction entre la liberté des Modernes et celle des Anciens dont il déduit «la nécessité du système représentatif». "Le système représentatif n’est autre chose qu'une organisation à l'aide de laquelle une nation se décharge sur quelques individus de ce qu'elle ne peut ou ne veut pas faire elle-même. Les individus pauvres font eux-mêmes leurs affaires; les hommes riches prennent des intendants [...]. Le système représentatif est une procuration donnée à un certain nombre d'hommes par la masse du peuple qui veut que ses intérêts soient défendus et qui, néanmoins, n’a pas le temps de les défendre toujours lui-

13. Slogan saint-simonien.

14. Eugène Buret, De la misère des classes laborieuses en Angleterre et en France, Paris, Paulin, 1840, 2 volumes, volume 2, p. 489. 
même $»^{15}$. Au sortir de la Révolution, l'idée de représentation est alors conçue par Benjamin Constant, comme l'expression d'une défense des intérêts de l'homme libre. Quant à François Guizot, il ne cesse, dans le même temps, de réduire la souveraineté du peuple à une illusion dont il attribue à Rousseau la responsabilité, ce dernier ayant "proscrit tout gouvernement fondé sur la représentation». De son point de vue, cette idée, si populaire, serait «destructrice de tout gouvernement, que dis-je de toute société » ${ }^{16}$. Une chimère qui jamais ne sera appliquée. L'origine du pouvoir repose sur la capacité des hommes à l'exercer. C'est pourquoi la représentation du souverain n'est pas pensée comme une délégation mais prend effet uniquement dans un gouvernement représentatif. «Il se propose précisément d'établir entre la société et le pouvoir, leur relation naturelle et légitime, c'est-à-dire d'empêcher que le pouvoir ne demeure, en droit, où il n'est plus en fait ${ }^{17}$. Dans l'esprit des autorités, et bien au-delà, on le sait, la vision de François Guizot l'emporte, y compris dans l'historiographie. Bien que mis à l'écart des sphères du pouvoir après 1848 , l'homme de gouvernement n'en demeure pas moins fidèle aux années fondatrices de la pensée libérale. Hostile à toute idée démocratique, au sens ancien du terme, conscient de rendre compte d'un pouvoir, qu'il qualifie lui-même de despotique, son admiration pour Napoléon, qu'il partage avec ses contemporains, est d'abord fondée sur une conception très restrictive de la souveraineté. À ses yeux, la France démocratique, celle des gouvernements représentatifs, «doit beaucoup à l'Empereur». Bien qu'il ait méconnu quelques-uns des besoins essentiels de notre temps, «il a rétabli au sein de la France démocratique, l'ordre et le pouvoir. Il a cru et prouvé qu'on pouvait servir et gouverner une société démocratique sans condescendre à tous ses penchants; c'est là sa grandeur ${ }^{18}$. On comprend mieux alors le point de vue des doctrinaires, présenté en défense de la Charte nouvellement acquise et que développe à la tribune de la Chambre Royer-Collard. Lecteur de Rousseau, celui-ci donne à comprendre mieux que personne le sens réel de la représentation politique. À la différence de Benjamin Constant, homme des Lumières, théoricien plus que praticien, le député libéral dit autrement et selon une conception opposée à celle de Rousseau, mais dans un sens identique, ce que représentation veut dire. "Il faut fixer invariablement le sens du mot représentation. Sans doute, lorsqu'on dit que la Chambre est

15. Benjamin Constant, «De la liberté des anciens comparée à celle des modernes. Discours prononcé à l'Athénée royal de Paris en 1819", in Benjamin Constant, De la liberté chez les Modernes. Ecrits politiques, textes choisis, présentés et annotés par Marcel Gauchet, Paris, Hachette, collection Pluriel, 1980 , p. 512.

16. François Guizot, «De la souveraineté personnelle», in Histoire de la civilisation en Europe (1828), depuis la chute de l'Empire romain jusqu'à la Révolution française, suivie de Philosophie politique : de la souveraineté, édition établie, présentée et annotée par Pierre Rosanvallon, Paris, Hachette, collection Pluriel, 1985, p. 363-364.

17. François Guizot, Des moyens de gouvernement et d'opposition dans l'état actuel de la France, intro-

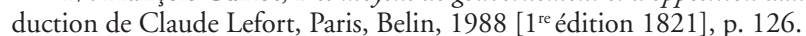

18. François Guizot, De la démocratie en France, Bruxelles, Wouters, 1849, p. 28. 
représentative, on entend que les intérêts, les droits, l'opinion, la volonté de la nation sont représentés dans cette Chambre et par conséquent la nation elle-même; car c'est là toute la nation politique. Maintenant qu'est ce que représenter une nation? Comment une nation peut-elle être représentée? Le mot représentation est une métaphore. Pour que la métaphore soit juste, il est nécessaire que le représentant ait une véritable ressemblance avec le représenté; et pour cela, il faut, dans le cas présent, que ce que fait le représentant soit précisément ce que ferait le représenté [...], je ne sais s'il existe sur terre ou même s'il peut exister un gouvernement parfaitement représentatif [...], la Chambre, telle que la Charte l'a conçue, est un pouvoir et non une représentation $»^{19}$.

Dès la fin de la Révolution, en France comme en Angleterre, la notion de souveraineté nationale a remplacé la souveraineté populaire et, à la faveur des monarchies constitutionnelles, sous le régime des capacités et du suffrage censitaire, toute représentation réelle devient illusoire. Mais la pratique de la représentation, dans un dispositif gouvernemental pensé sous l'angle de l'exercice d'un pouvoir, reste et restera la métaphore énoncée doctement par un des plus éminents doctrinaires. François Guizot ne développe pas un autre point de vue et Alexis de Tocqueville, bien que partisan - contraint - de la démocratie, expose, en apparence, une conception plus complexe de la représentation; il n'en demeure pas moins partisan d'un "pouvoir social, supérieur à tous les autres", tout en prévoyant l'existence d'une force susceptible d'en «modérer sa marche $»^{20}$. Le théoricien de la démocratie juge impossible d'éduquer le peuple au même titre que l'aristocrate ou le bourgeois, car celui-là doit avant tout «s'occuper des soins matériels de la vie». Par manque de temps «libre», le peuple ne trouve jamais le temps de se livrer au travail de la connaissance. C'est pourquoi, il s'irrite, s'agite et s'aigrit. «Les institutions démocratiques réveillent et flattent la passion de l'égalité, sans pouvoir jamais la satisfaire entièrement. Cette égalité complète s'échappe tous les jours des mains du peuple au moment où il croit la saisir ${ }^{21}$. C'est ainsi que l'idée d'une représentation par le vote universel est une illusion car la mauvaise éducation du peuple le conduit à choisir des représentants médiocres. Or, si la représentation n'est pas conçue comme un pouvoir en exercice - qui serait confié aux éléments les plus éduqués -, l'absence de représentativité risque de conduire le pouvoir à l'arbitraire le plus dommageable. Au-delà de la métaphore, chère à Royer-Collard, l'opinion d'Alexis de Tocqueville, comme on le sait, a largement triomphé.

19. Pierre-Paul Royer-Collard, Discours à la Chambre, février 1817. Cf. également mon commentaire dans Michèle Riot-Sarcey, Le Réel de l'utopie. Essai sur le politique au XIXe siècle, Paris, Albin Michel, 1998, p. 91-92.

20. Alexis de Tocqueville, De la démocratie en Amérique, Paris, Gallimard, collection Folio, 1986 [1 $1^{\text {re }}$ édition 1835], 2 volumes, volume 1, p. 377.

21. Idem, p. 299-300. 
Pierre Rosanvallon fait le même constat, en restituant les propos des socialistes, adeptes d'une expression directe de la démocratie. En cette première moitié du XIXe siècle, «faire ses propres affaires» est une formule qui, écrit-il justement, commence à circuler. En cherchant à populariser la souveraineté effective, les penseurs de la modernité de ce temps-là se seraient prononcés pour une souveraineté sans gouvernement. En faisant appel aux critiques de Marx et de Proudhon, Pierre Rosanvallon en conclut que «les théories du gouvernement direct anticipent ainsi moins l'avenir qu'elles ne dessinent une des figures paradoxales du retournement de la démocratie contre elle-même", en favorisant "l'absolutisation du vote", c'est-à-dire en laissant entendre que la voix directe du peuple s'exprimait par l'exercice du suffrage, précisément ${ }^{22}$. Conclusion logique si l'historien limite son analyse aux textes qui, pour l'essentiel, se réferent à une souveraineté identifiée au pouvoir législatif et exécutif. De ce fait, le sens courant ne peut être dépassé. Or, les expériences souveraines, concrètes, en l'absence de la liberté d'exercer ses capacités politiques, disent, dans la pratique, bien autre chose que le simple désir d'une démocratie directe. Il s'agit de prendre en charge l'existence au quotidien et de maîtriser la chaîne du travail effectué, à la manière, bien comprise, de Villermé. Des canuts réclamant un tarif à Pauline Roland qui, de sa prison, en appelle au gouvernement direct des travailleurs après l'échec patent de la République démocratique et sociale aux élections d'avril 1848. Une souveraineté en acte qui logiquement aboutit au succès sans précédent des associations en 1848-1849. Une démocratie directe qui passe d'abord par une prise en charge de ses propres affaires. Mais le pronom possessif ne se rapporte pas ici à l'individualité de chaque propriétaire, il recouvre tout un collectif sans lequel l'individu ne peut rien faire. À la manière de l'Association des associations dont les statuts avaient été rédigés par Jeanne Deroin, en 1849. Au cœur de cette pratique souveraine, on retrouve, en quelque sorte, les craintes énoncées, en 1816, par Joseph Fiévée, lorsque, au sortir de la Révolution, le conservateur ultra se penche sur les risques encourus par l'énoncé du principe de souveraineté populaire. Témoin direct, il n'a pas encore oublié le sens concret de l'idée de souveraineté. Il répond implicitement à Rousseau, et affirme que la souveraineté du peuple est contraire à la constitution et de fait inapplicable. De son point de vue, «l'idée d'un contrat social primitif suppose un peuple déjà formé en association et travaillant cependant à se former en association». Or, comme le peuple n'a pas inventé la civilisation et donc ne peut remonter à l'origine, en substituant sa propre souveraineté à celle de Dieu, les pouvoirs comme les lois le précèdent. Il doit donc se plier à l'ordre existant. Et de conclure : «Aussi cette doctrine n'a-telle, pour la première fois, été mise en avant que dans l'espoir de légitimer des

22. Pierre Rosanvallon, La démocratie inachevée. Histoire de la souveraineté du peuple en France, Paris, Gallimard, 2000, p. 174 et 179 . 
révoltes : elle ne prouve rien que l'orgueil naturel de l'esprit humain a mis en avant [...]. Cette souveraineté, comme tout ce qui est faux, échappe à l'application; on ne l'a pas plutôt reconnue qu'on ne sait plus qu'en faire »³. La souveraineté du peuple est insaisissable. Néanmoins, si nous voulons restituer la diversité significative de l'idée de souveraineté, il nous faut aller au-delà du sens commun exposé, par exemple, par François Furet selon lequel le Second Empire serait l'achèvement par excellence de la démocratie ${ }^{24}$ - qu'il définit en ces termes dans Le passé d'une illusion: "J'entends ici le terme dans ses deux significations classiques; la première désigne un type de gouvernement fondé sur le libre suffrage des citoyens, la compétition périodique des partis pour l'exercice du pouvoir et des droits égaux garantis à tous; la seconde renvoie plutôt à la définition philosophique des sociétés modernes, constituées par des individus égaux et autonomes, libres de choisir leurs activités, leurs croyances et leurs types d'existence $\aleph^{25}$. D'un côté une vision normative de la démocratie réduite au gouvernement issu des élections par la médiation des partis, de l'autre le «libre» choix d'une vie déterminée par les hiérarchies sociales! Où est le lieu d'exercice du pouvoir souverain du citoyen?

Aujourd'hui, la démocratie est identifiée au dispositif représentatif jusqu'à l'oubli de la souveraineté. Idée désormais insaisissable quand on a perdu sa pertinence populaire. Toute la difficulté de l'historien se révèle dans le délitement du sens du concept. Dans le temps des monarchies constitutionnelles, des royautés instables, des pouvoirs contestés, le mot même devient imprononçable, si ce n'est pour en dénoncer la perversité, la vanité ou l'illusion. Subsiste cependant la mémoire, le souvenir et le désir de s'approprier une idée qui, un moment, a pu paraitre accessible. Ce devenir de la souveraineté est infini et ne peut s'exposer dans un concept qui, toujours, réduit son sens à ce qui est entendu par ceux-là seuls qui ont su conjuguer exercice du pouvoir et capacité de l'exercer. Conception qui ne peut s'appliquer qu'à une minorité d'hommes libres.

Loin d'être achevé, notre volume n'a que l'ambition d'une ouverture vers de nouvelles recherches sur les expériences qui, partout dans le monde, se sont multipliées. Expériences fugaces, normalisées, difficiles à identifier mais qui, pourtant, disent comment la fraction de la population qui, le plus souvent, a été parlée par d'autres, a su un temps, même court, se rendre libre en luttant contre les tutelles qui la réduisaient au silence, ou ne lui permettaient

23. Joseph Fiévée, Histoire de la session de 1816, Paris, Le Normant, 1817, p. 20-22.

24. François Furet, La Révolution, 1770-1880, Paris, Hachette, 1988, p. 451. «Cette Démocratie est triomphante sous le Second Empire, bien plus que sous aucun des précédents régimes : c'est un effet naturel du temps qui passe, c'est aussi une volonté expresse de Napoléon III, au moment même où il a privé les Français de liberté politique». Le paradoxe n'est qu’apparent. En effet, à l'époque de la rédaction de l'ouvrage, François Furet privilégiait le sens de démocratie défini par Tocqueville en termes d'égalité civile dont les femmes étaient exclues, cela va s'en dire.

25. François Furet, Le passé d’une illusion. Essai sur l'idée communiste au XX' siècle, Paris, Robert Laffont, 1995 , p. 39. 
que de reproduire le discours ambiant. Comme très vite l'ordre hiérarchique a su se reconstituer, ces moments d'exception tombent dans l'oubli collectif et ne resurgissent qu'à la faveur d'une autre occasion où le désir de se sentir libre s'insère, se glisse dans les interstices de l'ordre existant.

Comment ne pas nous référer à notre propre actualité pour justifier le bien-fondé de ce détour vers une histoire oubliée? N'est-ce pas le peuple tunisien, égyptien ou encore libyen, qui aujourd'hui redonne sens à l'actualité de la souveraineté que l'on croyait circonscrite à l'expérience des révolutions occidentales qui, interprétées d'un seul point de vue, ont laissé croire à nos gouvernants que les peuples d'Afrique n'étaient pas encore entrés dans l'histoire. Belle leçon pour l'historien qui devra effacer toute trace d'une vision univoque et linéaire du passé, et recourir enfin à l'expérience critique de ceux dont l'histoire a été déniée.

Michèle Riot-Sarcey est professeure à l'Université Paris 8-Saint-Denis 